\title{
La certificación de competencias de los voluntarios como herramienta para mejorar el empleo juvenil y promover el voluntariado
}

\section{Certifying volunteers skills as a tool to improve youth employability and foster volunteering}

Virginia Vizcaíno Candela' (D), Elvira Medina Ruiz' (D)

\begin{abstract}
Resumen
Desde hace años se habla del voluntariado como generador de competencias y habilidades que pueden ser valiosas para la sociedad en general y el mundo empresarial en particular. La adquisición de esas competencias por parte de las personas voluntarias tendría un impacto directo en la mejora de la empleabilidad, especialmente en los más jóvenes. Pero para ello, es necesario que las entidades se doten de los mecanismos necesarios para poder certificar que, en efecto, se han adquirido esas competencias. La presente investigación tiene como objetivo analizar la viabilidad del reconocimiento de las competencias adquiridas a través de la realización de actividades de voluntariado, abordando y comparando distintas experiencias, para concluir cuáles son los modelos más idóneos para los/las voluntarios/as más jóvenes, teniendo en cuenta el diseño, el número de competencias, el proceso evaluador o la participación empresarial en el proceso.
\end{abstract}

Palabras clave: competencia profesional, voluntariado, juventud, empleo.

\section{Abstract}

Volunteering has been talked about for years as a generator of skills and abilities that can be valuable for society in general and the business world in particular. The acquisition of these skills by volunteers would have a direct impact on improving employability, especially among the youth. But for this, it is necessary that non-profit organizations equip themselves with the necessary certifying tools to confirm that these skills have been acquired. The aim of this research is to analyze the viability of the recognition of the competences acquired through volunteering activities, approaching and comparing different experiences in order to determine which are the most suitable models for younger volunteers, taking into account the design, the number of competencies, the evaluation process or corporate involvement in these experiences.

Keywords: professional competence, volunteering, youth, employment.

Vizcaíno Candela, V. y Medina Ruiz, E. (2021). La certificación de competencias de los voluntarios como herramienta para mejorar el empleo juvenil y promover el voluntariado. Itinerarios de Trabajo Social, 1, 45-53. https://doi.org/10.1344/ its.voi1.32332

\section{Introducción.}

Según la Plataforma Estatal de Voluntariado (en adelante, PVE), en España hay alrededor de 2,7 millones de personas voluntarias, un $6,7 \%$ más que los datos recogidos el año anterior (Plataforma del Voluntariado de España, 2019). Además, según el Observatorio del Voluntariado, que forma parte de la PVE, se calcula que durante la pandemia provocada por el COVID-19 el número de voluntarios/as se ha triplicado, llegando a ser un total de 4,5 millones de personas. Pese a esto, España no es el de los países con un mayor nivel de participación voluntaria de Europa. Con una media (con respecto al total de la población) de 10,7 de participación en tareas de voluntariado formales e informales, está muy alejada de la media del continente que se sitúa en 19,3 (Eurostat, 2017). De ahí que siga siendo tan importante centrar los esfuerzos en la captación, pero también en la permanencia de las personas voluntarias y la búsqueda de estrategias dirigidas a aumentar y mejorar la motivación ya sea a través de fuerzas internas o externas (Plataforma del Voluntariado de España, 2019).

En cuanto al perfil mayoritario actual del voluntariado, no se diferencia mucho de otros años, se trata de mujer, de entre 26 y 35 años, que tiene estudios superiores y un estatus socioeconómico medio-alto (Plataforma del Voluntariado de España, 2019). Con respecto a la edad, es destacable que cerca de la mitad de los/las voluntarios/ as $(46,2 \%)$ tiene menos de 35 años, lo que coloca a los/las jóvenes como un capital humano valioso e imprescindible dentro del grueso de voluntarios/as. Sin embargo, al analizar la evolución de los datos por tramos de edad, se observa un descenso del porcentaje de la participación de voluntarios/as de 14 a 34 años. Esto genera cierta preocupación en el seno de las organizaciones, puesto que su descenso ahora puede ser un reflejo de una bajada de la participación en tramos de mayor edad en el futuro (Plataforma del Voluntariado de España, 2019).

No es desconocida la preocupación de muchas organizaciones por mantener a sus voluntarios/as puesto que muchas dependen de ellos/as para el mantenimiento de
ACEPTADO: 08/12/2020 PUBLICADO: 21/01/2021

'Universidad de Murcia, España.

అElvira Medina Ruiz. Facultad de Trabajo Social. Campus Universitario de Espinardo. Espinardo (Murcia). 30.100. emedinaruiz@gmail.com 
servicios y programas (Chacón y Vecina, 2002). Por eso, es muy importante el estudio de la satisfacción, la mo vacion y la permanencia de las personas voluntarias dentro de las organizaciones a fin de mejorar su intención de permanencia. En cuanto a los motivos para realiza voluntariado, la Uiltina encuesta publicada (Plataform censo en los factores extrínsecos a la acción voble descomo son aprender, conocer personas y el funcionamiento de la organización, adquirir experiencia, etc. Teniendo en cuenta la elevada tasa de desempleo juven
en España, que según la última Encuesta de Población Activa está casi en el $40 \%$ siendo en mujeres del 41,23\% (Instituto Nacional de Estadistica, 2020), implantar un sistema que certifique la adquisición de competencias través de actividades le volquirín de, no solo sería cum plir con lo que aparece en la Ley 45/2015 de Voluntariado realizar voluntariado, lo que sería altamente beneficioso para entidades y las personas voluntarias.

El estudio de las competencias ha sido abordado por ferentes disciplinas a lo largo de los años, sin embargo, pocos estudios lo relacionan con la adquisición a través de experiencias de aprendizaje no formales como es voluntariado. Como deciamos anteriormente, el derech al reconocimiento de competencias queda reflejado la Ley 45/2015 de Voluntariado, obligando asía las enridades del Tercer sector de Acción Social, principales herramientas y protocolos pertinentes para hacer realdad este derecho.

1.1. Competencias y desempleo juvenil.

Existen numerosas y variadas definiciones en torno texmino competencia y serfa no solo tedioso sino imposiconcepto de conpetas dada la polisemia del témino. intento de la psicología aplicada al mundo empresaria por establecer criterios que permitieran "objetivar" rables fáciles de observar, evaluar, seleccionar, formar o recompensar.

Según el Diccionario de la Real Academia de la Lengua Es pañola, se define el término competencia como la "capa cidad, aptitud, idoneidad o incumbencia para desarrollar una actividad"

Por otra parte, López Ramírez (2014, p. 38) plantea com competencia la capacidad de realizar una actividad ef y utilizarlos de maner oportuna y positives necesarios tiempo determinado con el fin de llegar a la solución de los verdaderos problemas. Siguiendo esta misma línea, Spencer \& Spencer (1993) hacían referencia al término competencia como característica implícita en la persona y relacionada con el desempeño y la efectividad en un trabajo o situación.

Teniendo en cuenta esto, es posible inferir una sencilla fórmula para definir el concepto de competencia. El de sarrollo de una competencia implica la combinación de determinados. El término y acticudes en contextos cimiento + habilidad + actitud)

De hecho, según González y Larraín (2005) las compeDencias se desarrollan a traves de experiencias de aprenbaje integrando tres tipos de saberes: conceptual (sa(saber cer). (a)

más, según Tejada (2011) es posible evaluar la competencia a partir de los distintos tipos de saberes. Las dimensiones de una competencia componen un triánnón reciproca de manera que, tener una cosestencia no implica únicamente la pose a pirsontos, también refleja la aptitud o habilidad de persona y la actitud que presenta en un determinado contexto.

concepto de competencia es variado, según el ángulo pero el más extendido y aceptado es el ote saber bacer en un contexto determinado del campo científico que más se ha dedicado a su estudio, el de la educación.

os primeros escritos que se conocen al respecto de las competencias se remontan a escritos literarios relacionados con la educación, pese a que su resurgimiento se relaciona con la psicología del trabajo. En 1996 sale a la luz un informe de la Comisión Internacional sobre la cín de las Naciones Unidas promovido por la Organizala Cultura (UNESCO). Este informe plantea la reformulación de los distintos niveles educativos, con el fin de anclar los pilares claves para una educación de calidad a lo largo de la vida. En él, ya se apuesta por nuevas formas de certificación de competencias adquiridas a lo largo de la vida en ámbitos diversos (Delors, 1996, p. 36).

Desde finales de los 90 , se percibe una preocupación general por hacer reformas educativas y las aportaciones cadémicas van referidas, sobre todo, a la búsqueda de

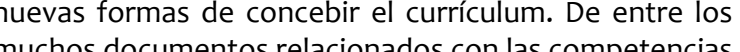
este periodo, destaca el denominado Definición y Selección de Competencias (en adelante, DeSeCo). Este es el nombre del proyecto de la Organización para la Cooperación y el Desarrollo Económicos (OCDE), encargado de definir y seleccionar las competencias consideradas esenciales para la vida de las personas y el buen funcionamiento de la sociedad. El fin ultimo del proyecto era desarrollar un sistema educativo integral mediante aquelas competencias que pueden considerarse básicas, llaLas primes escritos que

cospecto de cionados con la educación, pese a que su resurgimiento se relaciona con la psicología del trabajo. En 1996 sale a la luz un informe de la Comisión Internacional sobre la Educación para el Siglo XXI promovido por la Organización de las Naciones Unidas para la Educación, la Ciencia y la Cultura (UNESCO). Este informe plantea la reformuación de los distintos niveles educativos, con el fin de anclar los pilares claves para una educación de calidad a de certificación de enel, ya se apuesta por nuevas formas la vida en ámbitos diversos (Delors, 1996, p. 36).
Desde finales de los 9o, se percibe una preocupación general por hacer reformas educativas y las aportaciones académicas van referidas, sobre todo, a la búsqueda de nuevas formas de concebir el currículum. De entre los
muchos documentos relacionados con las competencias muchos documentos relacionados con las competencias
en este periodo, destaca el denominado Definición y Seen estén periodo, destaca ed lección de Competencias (en adelante, DeSeCo). Este es peración y el Desarrollo Económicos (OCDE) encargado de definir y seleccionar las competencias considerada esenciales para la vida de las personas y el buen funcionamiento de la sociedad. El fin último del proyecto era desarrollar un sistema educativo integral mediante aque llas competencias que pueden considerarse básicas, Illmadas competencias claves.

El Proyecto DeSeCo define competencia como "la cap cidad de responder a demandas complejas y llevar a cabo "supone una combinación de habilidades prácticas, conocimientos, motivación, valores éticos, actitudes, emociones, y otros componentes sociales y de comportmiento que se movilizan conjuntamente para lograr un acción eficaz" (Álvarez et al., 2008, p. 21).

En España, además tenemos otros antecedentes que nos han servido para marcar el camino, como fue la Ley Orgánica 5/2002, de 19 de junio, de las Cualificaciones de la Formación profesional y posteriormente el Real D competencias profesionales adquiridas por experien laboral, que tiene por objeto establecer el procedimien to y los requisitos para la evaluación y la acreditación de las competencias profesionales adquiridas por las personas a través de la experiencia laboral o de vías no forma les de formación, así como los efectos de esa evaluación y acreditación de las competencias.

Como remarca Gonzalez (2015) todas estas compete cias clave están asentadas en unas bases, cuyo objeto principal no es solo conocer su aplicación en la escuela competencias deben de poder aplicarse en multitud de contextos, abarcando miltiples áreas de la vida yomen tar el pensamiento crítico.

voluntariado es, sin duda, una buena opción para de sarrollar dicho pensamiento crítico, debido al impacto de una nueva cultura, un nuevo lenguaje y unas creencias a las que se ven sometidos habitualmente los/las voluntarios/as que participan en determinados proyectos (ConASDE Scouts España y Didnes Don Bosco de España ASDE Scouts España y Didania Federación de Entidades contribuyen al desarrollo persona y humano de los/las voluntarios/as, ya que se incrementa de forma notable la madurez, la confianza en uno mismo (en sus capacida des para resolver cualquier situación) y la independencia (Powell \& Bratovic, 2007).

En los últimos años, differentes experiencias han intentado relacionar competencias con voluntariado y la ma yoría coinciden en que la labor voluntaria contribuye la adquisición de las siguientes competencias: responsabilidad, analizar y resolver problemas, iniciativa y autode proyectos, creatividad, gestión del tiempo, capacidad de aprender, capacidad de enseñar, flexibilidad y adapta ción al cambio, impacto e influencia, capacidad de negode motivar, flexibilidad y adaptación al cambio, entre otros (Cook \& Jackson, 2006; Poyatos, 2013).

El interés surgido en la última década por vincular compeción de paliantar las elevado ha sido un reflejo más de la intenmente duras entre la población más joven Recordemos que, en 2013, España alcanzaba una cifra récord de paro juvenil, rozando el 57\% (Instituto Nacional de Estadística, 2014). La situación fue mejorando hasta situarse en un 30,5\% a finales de 2019 (Instituto Nacional de Estadístca, 2019), pero la actual crisis económica derivada de emergencia sanitaria provocada por el coronavirus $\mathrm{h}$ disparado de nuevo el paro entre los/las jóvenes. A fina el país con más tasa de desempleo entre los las meña como de 24 años según la Organización para la Cooperación y el Desarrollo Económico (OCDE). Nuestro país supera en la clasificación a países de nuestro entorno como Portugal (con una tasa de desempleo juvenil del $26,3 \%$ ), Italia (31,1\%) o Francia (19,7\%) (OCDE, 2020).

Los expertos apuntan que las consecuenclas sobre empleo dependerán mucho del nivel formativo, hecho que ya pudimos comprobar en la crisis económica de 2008 y de la que todavía muchos siguen sufriendo las nerables en esta ocasión pues sufren en mayor medidas contratos temporales precarios e inestables vinculados principalmente al turismo y la hostelería, las dos áreas más castigadas a causa de la pandemia (INJUVE, 2020). Según el estudio realizado por el INJUVE en el primer trimestre de 2020 en relación a las consecuencias socioeconómicas de la covid-19 sobre la población joven en

sitúa en el $25,2 \%$ de la pablación joven en España se sitúa en el $25,2 \%$ durante las primeras semanas del más de dos veces superior al que se ha dado entre la población de 30 a 64 años.

- Las personas jovenes son el colectivo con un mayor dientes de regulación de empleo, los conocidos como ERTE. Los y las jóvenes afectadas por un ERTE, aunque mantienen su relación laboral con la empresa, enfrentan un riesgo específico y grave de engrosar las - nil de dos mad laboral amenaza a la población juveprimeros en ser despedidos al término de los expe dientes de regulación de empleo; y a medio plazo, los que conserven sus empleos serán los más expuestos al despido si se materializa la amenaza de una crisis económica provocada por el Coronavirus.

Buscar alternativas para mejorar la empleabilidad juven se convierte en una tarea acuciante para todos los actores implicados. La certifcación y acreditación de compesa a la hora de optar a un empleo. Pero una opción valiocompetencias no siempre tiene que venir de un sisten educativo formal $y$ reglado, sino que existen diferentes 
opciones. Entre ellas, las labores de voluntariado se convierten en una alternativa atractiva, valiosa y útil para colectivo juvenil.

2. El voluntariado como generador de competencias.

La acción voluntaria y solidaria viene acompañándonos desde hace cientos de años y está presente en todas las magnífica manifestación de la solidaridad y la participa magnifica manifestación de la solidaridad y la participa
ción social de la ciudadanía que toma consciencia de su responsabilidad para con la sociedad en la que viven,
realizando durante su tiempo libre algún tipo de servicio realizando durante su tiempo libre algún tipo de servicio a la comunidad, cuyo objetivo principal es despertar y
generar la propia capacidad de las personas para moviligenerar la propia capacidad de las personas para movili-
zarse en la solución de sus problemas (Espinoza Vergara, zarse en

El voluntariado tampoco es algo nuevo en España, donde se viene hablando de acciones coordinadas y organla democracia, se generó el caldo de cultivo para su regulación materializándose en una Ley Estatal de Voluntariado en 1996 y posteriormente, en leyes autonómicas. La última reforma de la Ley de Voluntariado se produjo en 2015, un hecho que venían demandando durante años las entidades sociales, puesto que el propio fenómen Hoy en d́́ ya nadie niega su importancia e incluso. numerosos estudios que calculan su valor económico dentro de un país (Sajardo y Serra, 2008). Pero el voluntariado ha evolucionado en los últimos años, adquiriendo unas dimensiones nuevas debido al gran peso que tiene en las organizaciones sociales, evolucionando tambie las propias labores y funciones del voluntariado. De propia evolución incremento de la presencia voluntaria también ha aumentado la preocupación por su perm nencia y estabilidad dentro de las organizaciones (Dávila, 2008) ya que muchas de ellas requieren de su apoyo para
seguir adelante.

Pra definir voluntariado podemos acudir a la propia Ley

El conjunto de actividades de interés general desarrocladas por personas físicas, siempre que esta actividad
cumpla con las siguientes características: carácter soldario, realizada libremente, sin contraprestación económica o material (sin perjuicio del abono de los gastos reembolsables que el desempeno de la acción voluntaria ocasione a los voluntarios), desarrollada a traves de entidades de voluntariado con arreglo a program tos (art. 3).

Entre los numerosos aportes que trajo la reforma de Ley los derechos y deberes de los voluntarios siendo amplados y quedando mejor definidos. Es aquí donde queda recogido el derecho a "obtener reconocimiento de entidad de voluntariado, por el valor social de su contribución y por las competencias, aptitudes y destrezas adquiridas como consecuencia del ejercicio de su labor de voluntariado" (art. 10) nadas competencias personales, que además de ser im- portantes a nivel personal, pueden ser muy valiosas para pociedad en general, pero especialmente para empleadores. Son competencias relacionadas con la capacidad de escucha, la comunicación, la empatía, la autonomía, el rabajo en equipo o la responsabilidad.

quedar recogido en la Ley, las entidades tienen la oblficior de ofrecer a los voluntarios la posibilidad de certipor tanto, las competencias adquiridas a ráz de su abor voluntaria. Por este motivo, nos preguntamos qué sistemas existen para llevar a cabo este cometido, qué diferencias existen entre ellos y cuál sería el más apropiado para los jovenes.

o olvidemos que, además, la certificación de competencias se puede convertir en un potente incentivo que pue(Chinman \&Wandersman, 1999).

\section{Objetivos.}

Hia vez evidenciada la importancia de la gestión y certilas competencias a través de actividades de voluntariado para mejorar entre otras cosas, la empleavestigación con los siguientes objetivos.

objetivo general: analizar la viabilidad del reconocimiento de las competencias adquiridas a través de la realización, se actividades de voluntariado. Para su consecuy comparar distintas experiencias piloto en las que se acreditan las competencias adquiridas por el voluntariado; b) Evidenciar cuál de las experiencias es la mejor forma de acreditación de competencias pensando, sobre todo, en el voluntariado juvenil.

\section{Metodología.}

Para responder al objetivo de estudio y dada su naturalezán exploratoria, se ha optado por un diseño de investigaviabilidad o no del reconocimiento de las competen cias adquiridas mediante la realización de labores de voluntariado, así como los mejores modelos para ello. Para alcanzar la consecución de objetivos, se ha realizado una revisión bibliográfica y un estudio de las páginas web de las entidades implicadas y se han analizado los modelos de certificación de competencias vigentes para personas con nacionalidad española dentro y fuera del pais. A la hora de analizar los modelos se han tenido en cuenta variables como aprendizaje formal y no formal, competencias certifcadas, participación empresarial en y/o el ámbito de aplicación.

\section{Resultados}

A continuación, se presentan los principales modelos de certificación de competencias que siviven para certificar las competencias relacionadas con las labores de volu tariado.

https://doi.org/10.1344/its.voii.32332
5.1. Youthpass.

Youthpass, pasaporte de la juventud, en castellano, es miento y validación del aprendizaje no formal adquirido mediante la participación en el programa Juventud en Acción. Dicho programa tiene como objetivo promover
la ciudadanía activa de los y las jóvenes. A través del certificado Youthpass, la Comisión garantiza que la experiencia de aprendizaje adquirida sea reconocida como formal. Esta herramienta también es válida para los programas dentro del marco de Erasmust, en concreto para Intercambios Juveniles, Servicio Voluntario Europeo (SVE) y para la movilidad de personas que trabajan en ámbito de la juventud.

Youthpass permite certificar ocho competencias clave, entre ellas, la comunicación en lenguas extranjeras. Se gún un estudio de Taru \& Kloosterman (2016) sobre el impacto de este modelo, el creciente número de usuario de la juventud y que ha contribuido de diversas maneras al reconocimiento del aprendizaje formal y no formal. Las personas participantes consideran que Youthpass es un instrumento útil de cara a la inserción laboral, sin embargo, desean que sea reconocido formalmente por los sistemas, instituciones $u$ organizaciones nacionales en ámbito de la educación formal y en el mercado laboral. Sancristóbal (2015) manifiesta la importancia de generalzar este tipo de certificación a todo tipo de educación no trasladara a más programas europeos, promoviendo tuaciones de sensibilización a las administraciones y a las empresas, para la puesta en valor y conocimiento sobre las aportaciones del certificado Youthpass.

5.2. Europass.

Otra herramienta similar al Youthpass es el Europass, se gún su web oficial, el Europass o Pasaporte Europeo de
Competencias, es un portafolios compuesto por cinco Competencias, es un portafolios compuesto por cinco competencias y cualificaciones de la persona Este porta folios contiene el currículum y el Suplemento al Título de Técnico o Certificado de Profesionalidad.

El objetivo es ayudar a los ciudadanos a presentar sus competencias de manera eficaz, permitiéndoles así encontrar trabajo y formación dentro de las fronteras eropeas apostando, de nuevo, por la movilidad intercontnental. Según Conzález y Moreta (2013) el Europass tien su origen en la Decisión $\mathrm{n}^{\circ} 2241 / 2004 /$ CE del Parlamento Europeo y del Consejo de 15 de diciembre de 2004, rela de las cualificaciones y competencias.

cias adquiridas mediante aprendizaje no formal, pero siempre ligados al aprendizaje formal, pero existen otro modelos creados expresamente para certificar compe tencias adquiridas mediante la labor voluntaria.

5.3. VOL+.

Es un programa de certificación de competencias obte nidas a través del voluntariado que nació en 2014 y es impulsado por la PVE, precisamente para cumplir con lo . obligación de las entidades de voluntariado.

Este modelo ofrece la posibilidad de certificar hasta un máximo de siete competencias realizando labores de vo luntariado. Estas competencias son: analizar y resolver ción interpersonal, flexibilidad e innovación, iniciativa autonomía, organización y planificación y trabajo en equpo. La posibilidad de certificar la tenencia de cualquiera de estas competencias puede resultar muy valiosa de cara a cualquier entrevista laboral, independientemente de la edad. De hecho, los objetivos esta certificación son: 1. Reconocer la incidencia del voluntariado en el desa rrollo de competencias de las personas.

2. Visibilizar ante el mercado laboral que la práctica voluntaria promueve el aprendizaje y el desarrollo de competencias.

rias

La adquisición de habilidades y competencias a raíz de una práctica voluntaria es un proceso lo suficientemente complejo para que necesite tiempo y compromiso. Por eso, el modelo de la PVE diferencia por niveles de compromiso, valorando solo las competencias avaladas por do tres niveles: bajo, intermedio y alto, certificando competencias que se encuentren como mínimo en el nive intermedio de compromiso.

La gestión de este modelo requiere la presencia de un técnico o responsable de la PVE para realizar un acompañamiento y seguimiento de la experiencia de la persona voluntaria. Este tutor es, además, el nexo de unión entre la organización en la que el voluntario lleva a cabo sus tareas y la entidad responsable de certificar las compeprocedimiento, este seguimiento no tiene por qué ser presencial pero sí será exhaustivo y periódico, junto una autoevaluación, y concluirá con la emisión de un certificado que atestigüe la adquisición de las competencias pertinentes.

Este modelo puede resultar ventajoso a las organizaciones puesto que las libera de la carga de hacer el seguimiento de la adquisición de las competencias de la personas voluntarias. Tengamos en cuenta que muchas de las organizaciones no tienen un/a responsable de vo voluntaria sisue la prondo uno de los retos pendiontes de las entidades del Tercer Sector (Medina Ruiz, 2016).

5.4. Proyecto Reconoce

Con el apoyo del Instituto de la Juventud (INJUVE), este sistema estáliderado por la Confederación de Centros Juveniles Don Bosco en colaboración con la Federación Didania y ASDE Scouts de Espana, y se basa en un sistem Estas entidades se han unido con el obietivo de raria. car el valor de la experiencia del voluntariado, sensibit- 
zando a la sociedad en general, $y$ al tejido empresaria en particular, sobre la importancia de las competencias $y$ habilidades adquiridas mediante labores y tareas volun tarias y el efecto que
empleabilidad juvenil.

empleabilidad juvenil. El proyecto Reconoce es más que un certificado de con petencias, pretende crear una red de entidades juventel proceso voluntario y en la inserción laboral de los/las jóvenes, ofreciendo cuatro vías para participar:

- Voluntariado. Gracias al sistema online de acreditación el voluntariado puede certificar sus compete cias, siempre que la entidad en la que realiza el voluntariado forme parte del proyecto.

- Entidades. Pueden ser acreditadoras o colaborado ras. Las entidades acreditadoras forman parte de Red de Entidades yse encargan de evaluar a sus volu Proyecto Reconoce.

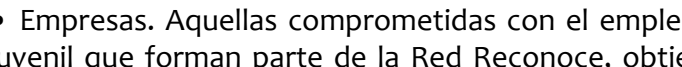
nen un distintivo de Empresa Reconoce, mejorando responsabilidad social corporativa y los procesos de selección de personal.

- Administración Pública. Todas las que se unen a Red Reconoce obtienen un distintivo. Estas admin traciones potencian la acreditación Reconoce en los mejora de la empleabilidad juvent

Como remarcábamos antes, el proyecto plantea un no vedoso sistema online de acreditación de la acción voluntaria que se puede gestionar desce cualquier dispositivo móvil u ordenador y mediante la APP creada exclusiva mente para gestionarlo. Las personas voluntarias que quieran acreditar su actividad solo deben registrarse solicitar la acreditación de alguna de las doce compete cias disponibles: negociación, tacto y prudencia, trabajo en equipo, comunicación interpersonal, fiablilidad téc ca y profesional, organización y planificación, liderar inmo, capacidad de aprendizaje, iniciativa y autonomí y analizar y resolver problemas. Estas doce competencia se pueden clasificar en tres grandes grupos: autogestión, organización y comunicación

Como sucedía en el modelo de la PVE, es necesario el gir al menos una persona como evaluador/a de competencias dentro de la entidad en la que se realiza la labor voluntaria. La persona evaluadora debe conocer bien organización, su estructura interna, el equipo de trabajo y llevar participando un mínimo de tres años en dich entidad y haber realizado un curso de formación MOO Massive Online Open Courses).

Según este sistema la persona evaluadora no tiene por que ser un trabajador/a de la entidad, sino que podría ser también una persona voluntaria que cumpliera los requsitos mencionados. Esto también es otra ventaja para las entidades, puesto que muchas no podrían asumir es cia de figuras de referencia para la gestión voluntaria. El proceso de acreditación de competencias del proyec- to Reconoce cuenta con tres fases: autoevaluación, evaluación y contraste.

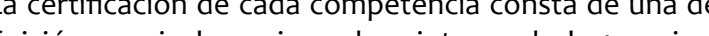

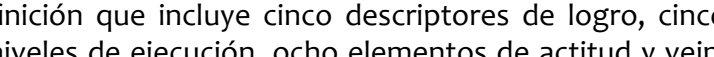
te dervacín y para los cuestionarios de covalua pán y au toevaluación. Una vez finalizado el proceso, la persona desde la APP. En dicho certificado se indica la definición le la competencia adquirida y el nivel que se tiene de la misma.

5. Talante SolidarioProyecto Reconoce.

Talante Solidario es una iniciativa creada por la Funda-

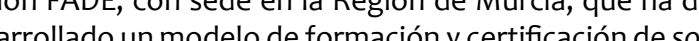
skills dirigida expresamente a personas voluntarias de entidades sociales, con la finalidad de mejorar el impacto de la propia acción social que realizan, todo gestionado a través de una plataforma digital.

Para Talante Solidario, la labor voluntaria de acción social tiene un impacto directo y visible en los colectivos tendidos, por lo que entienden se contribuye a la consucción de un mundo mejor. Esto se consigue tanto en ambito social como ellaboral, ya que las competencias mentan la empleabilidad y ayudan al desarrollo personal. Esta iniciativa además, sirve de punto de encuentro entre el Tercer Sector, el mundo profesional y la propia Administración Pública. Pese a tener sede en Murcia, este modelo está siendo utilizado por entidades de Madrid, Málaga, Valencia, Navarra y Galicia, además de otras con carácter estatal como CEPAIM o Fundación Diagrama, entre otras.

procedimiento de certificación se realiza a través de plataforma talantesolidario.org, en la que mediante competencias: liderazóo de servicio, análisis y resolucón de problemas, iniciativa y autonomía, empatía, comunicación interpersonal, organización y planificación, flexibilidad e innovación, trabajo de equipo, asertividad responsabilidad y compromiso. El procedimiento se estructura en diez pasos agrupados en teoria, ejercicios de entrenamiento y tests de evaluación. Es necesario ealizar un curso de ocho horas para poder certificar a destar alguna de las entidades asociades. Un tutoro tutora en mado expresamente para ello, será quien se encargue de compañar y certificar la competencia.

\section{Discusión.}

Desde hace unos años se han ido poniendo en marcha Diciativas piloto cuyo fin es identificar, $y$ en ocasiones también certificar, las competencias que se desarrollan a cialmente voluntariado, muchas de ellas pensadas espeOmo Holand (VPL), EScocia (VSkills) A ectos en paises teer Pass) o en España: VOL+ (lanzado en el 2014 por la
PVE, de la mano de Voluntariado y Estrategia y la Universidad de Alcalá), Reconoce (puesto en marcha en 2015) o más recientemente, Talante Solidario, coordinado por la Fundación FADE, además de los modelos comunes europeos Youthpass y Europass, identifican una serie de
competencias potencialmente desarrollables a través competencias po
del voluntariado.

Aunque el número y el tipo de competencias es diferente en cada modelo, sípodemos afirmar que la gran parte de las que se certifican a través del voluntariado suponen una mejora para el currículum de la persona voluntaria, puesto que son habilidades que las empresas buscan en los/las candidatos/as. De hecho, un informe realizado en 2013 por la multinacional Deloitte sobre el impacto de voluntariado en el empleo (Deloitte Volunteer Survey, 2013) concluía que el $81 \%$ de los directivos encuestado afirmaba que, en igualdad de condiciones, contrataría proceso de selección. En esta misma línea, Spera et a (2013) manifestaban que los/las jóvenes con experiencía en voluntariado tenín un $27 \%$ más de posibilidades $d$ encontrar un empleo que aquellos que no tenían dicha experiencia.

Para Sánchez et al. (2018) cuando los/las jóvenes estudiantes se integran en el ámbito laboral se les exige un determinado conocimiento técnico o competencias pro fesionales que le permitan desempeñar de forma óptima ras o hard skills. Pero para el desarrollo integral de una persona no basta solo con el conocimiento que pued aprender, es necesario un complemento de habilidades que le permitan tener autocontrol, confianza y trabaja en equipo. Se trata de las llamadas habilidades blandas o soft skills.

Los empleadores demandan profesionales competentes tanto en habilidades duras como blandas y la adquisició de las soft skills puede hacerse perfectamente a través de las experiencias de voluntariado (Cook \& Jackson de empresas y administraciones públicas en el diseño y proceso de acreditación en los modelos como Reconoce o Talante Solidario.

os años será avanzar en el estable cimiento de alianzas estratégicas con empresas, asociciones empresariales y asociaciones de profesionales $d$ recursos humanos, para que avalen el proceso de certifcación, y los propios certificados, dando reconocimien

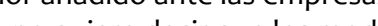

Esto no quiere decir que los modelos de alcance internasector empresarial, no sean positivos. De hecho se ha comprobado que las experiencias internacionales, como el voluntariado en otro país, proporcionan a la persona voluntaria oportunidades para reflexionar en sus propios valores, apertura a nuevas experiencias y el desarrollo $d$ conciencia de uno mismo e identidad propia, sobre todo si entra en contacto con culturas diferentes (Fee \& Gray, En cuanto a la idoneidad de los modelos analizados, $\mathrm{Vol}$ mente para ta certificaín de mediantela experienciavoluntariaycompart del catálogo de competencias, aunque el número sea diferente. Los tres han optado por evaluarlas mediante escalas de valor internedio, siendo los procesos metodológicos del modelo Reconoce y Talante Solidario más complejos y completos por su estructuración en fases: la autoevaluacion, informe del evaluador de la entidad contraste por parte del equipo auditor.

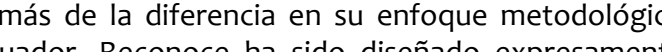
para el colectivo juvenil y, al igual que Talante Solidario, dispone de un soporte digital de acreditación, lo que priori puede resultar más fácil y atractivo para la población más joven.

\section{Conclusiones.}

Pese a que el voluntariado ha aumentado en los últimos y social, nada a la sticacion de encrenento se vaya a consolidar a corto plazo si tomamos de modelo la crisis anterior. Los niveles de participación solidaria y voluntaria en España están muy alejados de la media europea y tampoco son muchas las medidas puestas en marcha para modificar esta situación. Lo cierto es que una gra parte del tejido asociativo en España lleva muchos años nutriéndose del capital humano de miles de personas que donan tiempo, conocimiento y ganas para ayudar del voluntariado son personas jóvenes, pero en los úttmos años también se ha observado un descenso entre este segmento de edad (Plataforma del Voluntariado de España, 2019).

Los/las jóvenes son cada vez más dinámicos y manifiestan niveles más bajos de permanencia dentro de las organizaciones, a la vez que apuestan por el polivoluntariado y el ciberactivismo (Plataforma del Voluntariado de España, 2019; Folia, 2010). Hacer atractiva la labor voluntaria para inciarla o mejorar los niveles de satisfacción, deción ser una prioridad para entidades y administraciones los próximos años. La posibilidad de acreditar la adquisición de habilidades y competencias mediante labores de voluntariado no solo es una obligación de las entdades plasmada en la vigente Ley de Voluntariado sino que, además, puede ser un poderoso incentivo para los más jovenes que cada vez buscan mayores razones ex ternas que internas a la hora de iniciar labores de voluntariado (Plataforma del Voluntariado de España, 2019). las profesionales que trabajan en las entidades, sino que debe ser un compromiso y una prioridad estratégica de las direcciones y gerencias de las organizaciones, a igual que la generación de planes de formación para el voluntariado y la necesidad de disponer de profesionales adecuados para su gestión (Chacón y Vecina, 2002). En este sentido, el/la trabajador/a social, profesional de referencia en servicios sociales y pieza indispensable en el Tercer Sector, parece una de las opciones más idóneas (Medina Ruiz, 2018)

de las tasas mayores de pae España volveráa a sufrir una repercutirá en un aumento de la competencia a la hor 
de encontrar empleo, por lo que los empleadores busca rán elementos que distingan unos curriculums de otros. Como hemos podido comprobar, el reconocimiento competencias adquiritlas a través del voluntariado no solo es viable, sino que es un derecho de la persona voluntaria. Actualmente conviven diferentes certificados para recocer competenclas, pero son muchas las entque podrí estar contribuy ha a una vulneracionde los derechos del voluntariado.

La implantación de cualquiera de los certificados mencionados anteriormente en una entidad, supondría una mejora en la empleabilidad de las personas voluntarias un importante incentivo a la hora de realizar actividades de voluntariado (Confederación de Centros Juveniles Don Bosco de España, ASDE Scouts España y Didania Fe deración de Entidades (ristianas de Tlempo Libre, 2015). labor voluntaria dentro de la sociedad, otro de los retos pendientes, pero es cierto que, si pretendemos poner foco en el empleo juvenil, la opción preferible es la de Reconoce y Talante Solidario.

En los últimos años, muchas empresas y organizaciones sociales se han interesado por la certificación de compe tencias y la implantación de programas de voluntariado corporativo como parte de su politica de responsabilida social corporativa (Poyatos, 2013). Realizar voluntariado favorece la retención de los/las empleados/as jovenes sarrollo como capital humano en el mercado de trabajo (Babušytė, 2019).

Lo que parece evidente es que hacer voluntariado no solo nos beneficia como personas, sino que tiene efectos positivos sobre nuestra experiencia y vida labora. El voluntariado ha sido y es uno de los generadores democracia y de convivencia civica más valiosos (Arnanz, 2011) y si se cump
portante en los prox portante en los próximos años.

\section{Bibliografía.}

Álvarez, S., Pérez, A. y Suárez, M.L. (2008). Hacia un enfoque de la educación en competencias. Oviedo:
Consejería de Educación y Ciencia Dirección Cen ral de Políticas Educativas y Ordenación Académica - Servicio de Evaluación, Calidad y Ordenación Académica.

Arnanz, E. (2011). Voluntariado y participación. Revista Española del Tercer Sector, 18, 75-96.

(Coord) (2019).Voluntariado corporativo: in sas y mpleados. Bruselas: NCO Socialinis ve emp acón, F. y Vecina, M.L. (2002). Gestión del voluntariado. Madrid: Síntesis.

Chinman, M. \& Wandersman, A. (1999). The benefits an costs of volunteering in community organizations: luntary Sector Quarterly, 28(1), 46-64.

Cook, P. \& Jackson, N. (2006): Valuing Volunteering. Lo P.

ña, ASDE Scouts España y Didania Federacio
Entidades Cristianas de Tiempo Libre (2015). La situación del voluntariado juvenil ante el empleo: competencias y empleabilidad. Recuperado de https:// reconoce.org/estudio.pd

Dávila, M.C. (2008). Abandono del voluntariado. Tasas de abandono y causas más frecuentes. Comunicación Citte Volunteer Survey (2

-Impact Series. Washington: Deloitte Recuperadode www.deloitte.com/us

Delors, J. (Coord) (1996). La educación encierra un tesoro. Informe a la UNESCO de la Comisión Internaciona sobre la Educación para el Siglo XXI. Madrid: Santillana-Ediciones UNESCO.

Espinoza Vergara, M. (1988). El voluntariado: una ideología de compromiso: Bienestar Social, Servicios Socia-

ostat (2017). Participation in formal or informal voluneducational attainment level Last update: 14-11-2017.

Fee, A. \& Gray, S. (2007). Climb High, Sleep Low: The Unique Learning Environments of International Volunteer Placements. Sidney: University of Sydney.

olia (Franco y Guilló) - Observatorio del Voluntariado PVE (2010). Diagnóstico de la situación del voluntariado de acción social en Espana. Estudio y sistema de indicadores clave. Madidi. Ph. Estoma del Volunto-

nzález, J. (2015). Competencias básicas y el método scout: propuesta de intervención [Trabajo de Fin de
Grado, Universidad de Valladolid, Castilla y León]. Repositorio Documental de la Universidad de Valla dolid. http://uvadoc.uva.es/handle/10324/16288

González, L.E. y Larrain, A.M. (2005). Formación Universitaria Basada En Competencias: Aspectos Referenciales. Barranquilla. Cetro Interniversitario de Desarrollo.

González L. A. y Moreta, M. (2013). Estudio de las Escuelas Asociación Ser Joven.

Instituto de la Juventud y Consejo de la Juventud de Es. paña (INJUVE) (2020). Juventud en riesgo: análisis de las consecuencias socioeconómicas de la COVID-19 sobre la población joven en España. Primer informe. Recuperado de http://www.injuve.es

Instituto Nacional de Estadistica (2020). Encuesta de Población Activa, Primer Trimestre 2020.

列 blación Activa, Tercer Trimestre 2014.

elas 6(13), 35-48. Recuperado de http://www.edu-fisica. com

Medina Ruiz, E. (2016). El voluntariado en España: situa ción actual, tendencias y retos. La Razón histórica: Revista hispanoamericana de Historia de las Ideas,

33, 110-129.
Medina Ruiz, E. (2018). Gestión del voluntariado desde la perspectiva senior: una propuesta desde el trabajo
social. En E. Iglesias (Coord), Intervención para Ia social. En E. Iglesias (Coord), Intervención para la (103-120). Monterrey: Tirant Lo Blanch.
OCDE (2020). Unemployment Rates. News Release: July 2020.

Plataforma del Voluntariado de España (2019). Así somos en 2018. Retrato del voluntariado en España. Madric La Plataforma del Voluntariado-Observatorio de Voluntariado. Recuperado de https://plataformavomos-2018.pdf

Powell, S. \& Bratovic, E. (2007). The impact of long-term youth voluntary service in Europe: A review of puAVSO \& ProMENTE Social Research.

Poyatos, J. (2013). Desarrollo de competencias a través de Voluntariado Corporativo: Guía Voluntare. Madrid:

Voluntare.
Sajardo, A. y Serra, I. (2008). Avances recientes en la investigación sobre el voluntariado: valoración ecovoluntariado y voluntariado corporativo. CIRIEC-Es. paña, revista de economía pública, social y cooperativa, 63, 191-225.

Sánchez, O. M., Amar, R. M. y Triadú, J. X. (2018). Habilidades blandas: necesarias para la formación integral del estudiante universitario. Revista Cientifíca

Ecociencia, 5, 1-18.
Sancristóbal, L. C. (2015). El panorama de la validación y acreditación de competencias en España. Certiun Joun

Spencer, L.M. \& Spencer, S.M. (1993). Competence at
Work. Models for Superior Performance New York: John Wiley \& Sons, Inc.

Spera, C., Ghertner, R., Nerino, A. \& DiTommaso, A. (2013). Volunteering as a Pathway to Employment: Does Volunteering Increase Odds of Finding a Job for the Out of Work?. Washington, DC: Corporation for National and Community Service, Ofice of Re-

search and Evaluation.
Taru, M. \& Kloosterman, P. (2016). Youthpass impact study. Young people's personal development and Brussels: European Commission

Tejada Fernández, J. (2011). La evaluación de las competencias en contextos no formales: dispositivos instrumentos de evaluación. Revista de Educación, $354,731-745$ 